\title{
La paradiplomacia de los gobiernos subnacionales en América del Norte*
}

\author{
The paradiplomacy of subnational governments in North America
}

A paradiplomacia dos governos subnacionais na América do Norte

Recibido el 12 de julio de 2017. Aceptado el 2 de octubre de 2017

Roberto Zepeda Martínez**

México

\section{Resumen}

Para citar este artículo:

Roberto Zepeda Martínez (junio, 2018). La paradiplomacia de los gobiernos subnacionales en América del Norte. Ánfora, 25(44), 17-41. Universidad

Autónoma de Manizales. ISSN

0121-6538.
Objetivos: determinar los factores que han contribuido a la expansión de las actividades internacionales de los actores subnacionales y detectar los temas que predominan en las relaciones entre las provincias canadienses y los estados subnacionales de América del Norte. Asimismo, identificar las estrategias y mecanismos con los cuales se dan las relaciones entre estos gobiernos subnacionales. Metodología: la estrategia metodológica de tipo cualitativo comprende dos dimensiones: la primera, se enfoca en los factores

que explican estas dinámicas y en la revisión conceptual usada para hacer referencia a las actividades transnacionales de los gobiernos subnacionales en América del Norte, en particular la paradiplomacia. La segunda, escudriña información oficial, reportes y publicaciones de los gobiernos nacionales y subnacionales de la región. Posteriormente, se examinaron las actividades internacionales de tales gobiernos para determinar su naturaleza, características y objetivos. Resultados: se evidenció que las actividades

\footnotetext{
* Este artículo se deriva del proyecto: "Dinámicas subnacionales en América del Norte: relaciones de los gobiernos no-centrales de Canadá con sus contrapartes en Estados Unidos y México", realizado en al CISANUniversidad Nacional Autónoma de México, UNAM. Proyecto en curso, iniciado en septiembre de 2015.

** Doctor en Ciencias Políticas y Sociales. Investigador de Tiempo Completo del Centro de Investigaciones Sobre América del Norte (CISAN) de la Universidad Nacional Autónoma de México, UNAM. Correo electrónico: zepeda_roberto@hotmail.com
} 
internacionales de los gobiernos subnacionales están vinculadas con el desarrollo económico y con temas como medio ambiente, recursos naturales, desarrollo sustentable, seguridad, cultura, educación, ciencia y tecnología; se llevan a cabo a través de foros institucionales de deliberación y toma de decisiones, oficinas internacionales y acuerdos de cooperación. Conclusiones: los gobiernos subnacionales contribuyen a la gobernanza de América del Norte, a través de la formulación de políticas públicas regionales para afrontar retos comunes. Los acuerdos se circunscriben en las áreas de jurisdicción de los gobiernos subnacionales y, al mismo tiempo, revelan cierto grado de autonomía respecto al gobierno central.

Palabras clave: Paradiplomacia; Cooperación internacional; Políticas públicas.

\section{Abstract}

Objetives: to determine the factors that have contributed to the expansion in the international activities of the subnational actors and to identify the issues that prevail in the relations between the Canadian provinces and the subnational states of North America. Likewise, to identify the strategies and mechanisms that favor the relationships between these subnational governments. Methodology: the qualitative methodological strategy comprises of two dimensions: the first focuses on the factors which explain these dynamics and on the conceptual revision to refer to the transnational activities of subnational governments in North America, in particular the paradiplomacy. The second one examines official information, reports and publications from national and subnational governments in the region. Subsequently, the international activities of these governments were examined to determine their nature, characteristics and objectives. Results: it was highlighted that the international activities of subnational governments are linked to economic development and to issues such as the environment, natural resources, sustainable development, security, culture, education, science and technology. They are carried out through institutional deliberation and decision-making forums, international offices and cooperation agreements. Conclusions: subnational governments contribute to the governance of North America, through the formulation of regional public policies to face common challenges. The agreements are limited in the areas of jurisdiction of the subnational governments and, at the same time, they show/disclose a certain degree of autonomy with respect to the central government.

Keywords: Paradiplomacy; International cooperation; Public policies. 


\section{Resumo}

Objetivos: determinar os fatores que tem contribuído à expansão das atividades internacionais dos atores subnacionais e detectar as temáticas que dominam nas relações entre as províncias canadenses e os estados subnacionais da América do Norte. Além disso, identificar as estratégias e mecanismos com os quais as relações entre esses governos subnacionais são dadas. Metodologia: a estratégia metodológica de tipo qualitativo compreende duas dimensões: a primeira se enfoca nos fatores que explicam estas dinâmicas e na revisão conceitual usada para se referir às atividades transnacionais dos governos subnacionais na América do Norte, particularmente na paradiplomacia. A segunda, examina informações oficiais, relatórios e publicações dos governos nacionais e subnacionais da região. Posteriormente, as atividades internacionais de tais governos foram examinadas para determinar sua natureza, características e objetivos. Resultados: Evidenciou-se que as atividades internacionais dos governos subnacionais estão ligadas ao desenvolvimento econômico e a questões como o ambiente, recursos naturais, desenvolvimento sustentável, segurança, cultura, educação, ciência e tecnologia; estas são realizadas através de fóruns institucionais de deliberação e tomada de decisões, acordos de cooperação, e em escritórios internacionais. Conclusões: os governos subnacionais contribuem à governação da América do Norte, através do desenvolvimento de políticas públicas regionais para enfrentar os desafios comuns. Os acordos se circunscrevem nas áreas da jurisdição dos governos subnacionais e, ao mesmo tempo, revelam um grau de autonomia do governo central.

Palavras-chave: Paradiplomacia; Cooperação internacional; Políticas públicas 


\section{Introducción}

Desde el último cuarto del siglo XX, la creciente globalización económica ha generado desafíos sin precedentes que en la mayoría de los casos superan la capacidad de maniobra del Estado, llevando a una nueva forma de gobernanza. Como resultado, han surgido nuevos espacios de poder y toma de decisiones. En este contexto, los estados ${ }^{1}$ subnacionales han emergido como uno de los actores más relevantes del siglo XXI en la esfera internacional. Los grandes problemas globales no han sido resueltos desde los gobiernos centrales, pero encuentran cauces de solución en los gobiernos subnacionales.

A partir de la entrada en vigor del Tratado de Libre Comercio de América del Norte (TLCAN) en 1994, se han fortalecido sus relaciones comerciales. Los países de esta zona de libre comercio -México, Estados Unidos y Canadá-, producen cerca del $30 \%$ de los bienes y servicios en el mundo. El comercio en la región es al menos $265 \%$ más grande que hace dos décadas, cuando entró en vigor el TLCAN, mientras que la inversión se ha multiplicado por seis (Secretaria de Relaciones Exteriores [SRE], 2014). No obstante, no ha habido un progreso relevante en otras formas de integración. En otras palabras, la integración regional en Norteamérica ha sido relegada por los gobiernos nacionales de estos tres países. Sin embargo, las relaciones entre los gobiernos subnacionales se han expandido en diversas áreas desarrollando redes de colaboración, que son cruciales para comprender las dinámicas y realidades emergentes en la región.

Algunos autores han examinado la paradiplomacia de los gobiernos subnacionales en la región. Por ejemplo, las actividades internacionales como el comercio de las provincias canadienses con Kukucha (2009) o el rol de las provincias canadienses en cuanto a la implementación de tratados con Paquin (2010); además, el caso específico de las motivaciones de Quebec en su estrategia internacional que plantea Balthazar (1999). En el caso de los Estados Unidos, el rol de los gobiernos subnacionales como actores internacionales ha sido examinado por varios autores considerando la relevancia y características de sus dinámicas internacionales (Hocking, 1993; Fry, 2004; McMillan, 2012). Sin embargo, no se ha propuesto una perspectiva regional que considere las relaciones internacionales entre los gobiernos subnacionales de Canadá y Estados Unidos establecidas a través de diversos esquemas de cooperación.

De tal manera, surge el cuestionamiento sobre si el proceso de integración económica en América del Norte ha llevado al aumento de las relaciones

1. Para evitar confusión, en este artículo el término "estado" (en minúscula) se refiere a entidades federativas o unidades subnacionales de países con sistema federal, por ejemplo, los estados de México y Estados Unidos, así como las provincias de Canadá. Mientras que "Estado" (en mayúscula) se utiliza como sinónimo de país o “Estado-nación”, por ejemplo, México, Estados Unidos y Canadá. 
internacionales entre los gobiernos subnacionales de la región y de qué manera se han presentado estas tendencias en los países de la región.

\section{Metodología}

Este artículo recurrió a una estrategia metodológica de tipo cualitativo que considera dos dimensiones de observación y análisis. La primera, consiste en la revisión conceptual sobre enfoques utilizados en las ciencias políticas y relaciones internacionales para hacer referencia a las actividades transnacionales de los gobiernos subnacionales; en especial se recurre al concepto de paradiplomacia. Además, una revisión de los factores que explican estas dinámicas, tales como: federalismo, descentralización, globalización y gobernanza. La segunda parte consta del análisis pormenorizado de información oficial, reportes y datos de fuentes primarias, generados por los gobiernos nacionales y subnacionales de la región. Por ejemplo, las organizaciones regionales ${ }^{2}$ integradas por gobiernos subnacionales, pero también las páginas web oficiales de gobiernos subnacionales que dan cuenta de sus actividades internacionales. Asimismo, de la Secretaria de Relaciones Exteriores de México, y de la embajada de México en Estados Unidos.

El periodo de análisis se concentra de mediados de la década de 1980 al 2015 , debido a que en tal época diversos procesos como la globalización económica y la descentralización se intensificaron. Por ejemplo, la integración económica y las políticas neoliberales se acrecentaron a partir de la puesta en marcha del Tratado de Libre Comercio entre Estados Unidos y Canadá (1988), así como el Tratado de Libre Comercio de América del Norte (TLCAN) entre Estados Unidos, Canadá y México. La estrategia de análisis se concentra en las relaciones internacionales de las provincias de Canadá con los gobiernos subnacionales en Estados Unidos.

\section{Resultados}

Diversos factores han contribuido a la expansión de las relaciones internacionales de los gobiernos subnacionales en América del Norte, en

2. Por ejemplo, la Conferencia de Gobernadores y Premieres de los Grandes Lagos y San Lorenzo (CGLSLGP), la Alianza entre estados del Sudeste de Estados Unidos y las Provincias Canadienses (SEUSCP), entre otras. 
particular entre los gobiernos subnacionales de Canadá y sus contrapartes en Estados Unidos. En la primera sección de estos resultados se muestran algunos aspectos clave del federalismo, en particular los niveles de descentralización en los países de América del Norte. La segunda sección se concentra en las características, dinámicas, y actores más relevantes de la gobernanza, así como la fragmentación del Estado, donde se presenta la expansión de nuevos centros de poder y autoridad.

De igual manera, se evidencia cómo la globalización neoliberal ha impactado en los gobiernos subnacionales, $\mathrm{y}$ ha derivado en procesos como la internacionalización subnacional y la emergencia de acuerdos de gobernanza regionales o transnacionales. Finalmente, se muestra el concepto de paradiplomacia con sus diversas formas y características, en particular en Norteamérica. En la tercera sección, se discute la dimensión de la paradiplomacia de las provincias canadienses y los estados de Estados Unidos. Se evidencian los casos transfronterizos entre los estados subnacionales de la región, creando foros permanentes que en algunos casos se han institucionalizado.

Posteriormente, se muestran y discuten las dimensiones de la paradiplomacia de las provincias canadienses, destacando los factores que propician estas tendencias. Las actividades de paradiplomacia en la región se han incrementado como resultado de la globalización económica, pero han sido más evidentes en países con altos niveles de descentralización que han otorgado mayor influencia y margen de maniobra a los actores subnacionales en el campo internacional.

Se ha detectado una expansión de las relaciones internacionales entre los gobiernos subnacionales en América del Norte durante las últimas dos décadas, la cual está vinculada con las actividades económicas y comerciales de la región subnacional, pero también se deriva de la cooperación transnacional en temas tan variados como medio ambiente, recursos naturales, desarrollo sustentable, seguridad, cultura, así como educación, ciencia y tecnología. La mayoría de los acuerdos y políticas públicas que se han concretado se circunscriben a las áreas de jurisdicción subnacional y, al mismo tiempo, revelan cierto grado de autonomía respecto al gobierno central.

En algunos casos, los gobiernos subnacionales persiguen objetivos independientes de los gobiernos nacionales, en especial aquellas provincias que se consideran como una nación dentro de un Estado, como por ejemplo el caso de Quebec en Canadá, aunque en años recientes esta tendencia ha disminuido. Las acciones internacionales de los gobiernos subnacionales contribuyen a solucionar problemas de la región y al mismo tiempo hacen frente los problemas globales que no han sido solucionados desde los ámbitos nacionales. En la gobernanza de la región, destaca la participación de los gobiernos subnacionales en espacios de cooperación transnacional con otras unidades subnacionales, que en ocasiones 
se convierten en foros institucionales, con el fin de coordinar los esfuerzos para enfrentar desafíos comunes en diversas áreas.

Una conjunción de factores ha contribuido a la expansión de las actividades internacionales de los gobiernos subnacionales. Se considera que los gobiernos subnacionales tienen un amplio margen de maniobra en países con sistemas federales, con un alto nivel de descentralización. Además, la globalización neoliberal ha erosionado el poder del Estado-nación y han surgido nuevos actores en el sistema internacional. Lo anterior ha derivado en una nueva forma de gobernanza con una multitud de actores, entre los que destaca el rol de los gobiernos subnacionales, los cuales han formado redes de interacción con sus contrapartes en una misma región, y de países distantes. La siguiente sección se enfoca en estos factores.

\section{Federalismo y descentralización}

Es relevante examinar las características del federalismo, ya que, en algunos países con este sistema, los gobiernos subnacionales tienen una amplia capacidad para formular políticas públicas en varias áreas, incluyendo la realización de actividades internacionales. Los tres países de Norteamérica tienen sistemas federales de gobierno, pero cada país presenta distintos niveles de descentralización entre gobiernos centrales y no-centrales. Fry (2004, p. 4) observa que Canadá tiene el sistema más descentralizado, con los gobiernos de las 10 provincias ejerciendo mayores atribuciones en la formulación de políticas públicas, comparado con las que tienen los 50 estados de los Estados Unidos o los 32 estados de México. Además, la evolución histórica del federalismo es diferente en cada país, especialmente en el caso de México, donde ha predominado una tendencia centralista en la vida política del país. Canadá puede ser considerado como el país con uno de los sistemas federales más descentralizados del mundo (Hague y Harrop, 2005, p. 232).

El surgimiento y desarrollo histórico del federalismo difiere entre los países de la región. Por ejemplo, Estados Unidos y Canadá surgieron como países formados por gobiernos subnacionales que contaban con cierta autonomía respecto al gobierno central, mientras que en el caso de México el federalismo se creó desde un gobierno centralista (Curzio, 2000; Márquez-Padilla, 2012) .

\footnotetext{
3. Para más sobre las características del federalismo en Estados Unidos, véase: Paz-Consuelo MárquezPadilla (2012), Justicia Internacional: ideas y reflexiones, México, CISAN-UNAM. Respecto al caso de México, véase: Leonardo Curzio (2000), "El federalismo mexicano: balance y perspectivas", en Guillermo Ibarra y Ana Luz Ruelas (eds.), México y Canadá en la globalización: fronteras tensionadas en América del Norte, México: Porrúa, pp. 257-280.
} 
Canadá se caracteriza por tener un federalismo multicultural, multinacional y bilingüe (Burgess, 2006: 120); es decir, Canadá es un país muy diverso, donde se reconoce tal diversidad del gobierno, lo que explica el alto grado de descentralización y autonomía de las provincias.

De acuerdo con el Programa de las Naciones Unidas para el Desarrollo (PNUD), la gobernanza descentralizada, cuidadosamente planeada, implementada de manera efectiva y gestionada adecuadamente, puede llevar a una mejora notable en el bienestar de las personas en el nivel local y su efecto acumulado puede conducir al fortalecimiento del desarrollo humano; sin embargo, la gobernanza descentralizada no es una panacea ni tampoco una solución rápida $(2004$, p. 2). Este mismo organismo asegura que la clave para una gobernabilidad descentralizada favorable al desarrollo humano es asegurar que las voces y demandas de los pobres, especialmente las mujeres, contribuyan a su diseño, ejecución y seguimiento. Asimismo, Fry (2011) argumenta que los gobiernos subnacionales son más receptivos a las necesidades de los ciudadanos y están más adaptados a sus problemas; por lo cual, trasladar responsabilidades a niveles más bajos de gobierno puede contribuir a una formulación de políticas públicas más efectiva y eficiente.

En Canadá las provincias administran los sistemas de salud, educación, prestaciones sociales, trabajo, entre otros rubros (Véase cuadro 1). En 2011, alrededor del $47 \%$ del gasto público se concentró en el nivel provincial en Canadá (el mayor porcentaje en este rubro entre países de la OCDE), mientras que el gobierno local concentró $20 \%$ y el gobierno federal, un $28 \%$ del gasto público (OCDE, 2015). Estas facultades de las provincias les permiten diseñar la implementación de tratados internacionales que afecten los rubros bajo su jurisdicción (Paquin, 2010). A su vez, Hale (2012) evidenció que una serie de decisiones judiciales desde el siglo XIX les ha dado, significativamente, a las provincias mayor jurisdicción y discreción en áreas de política pública que van desde el desarrollo económico a la regulación de las relaciones laborales, mercados de capital, y desarrollo de recursos naturales.

Para Kincaid (2013, p. 111), los 50 estados de Estados Unidos tienen facultades limitadas para realizar actividades internacionales derivadas de tres factores: 1) su autoridad constitucional para involucrarse en la arena internacional en forma limitada como estados, pero no como Estados-nación; 2) su libertad política para perseguir intereses estatales y locales a nivel internacional; 3) su capacidad gubernamental para actuar de manera independiente en el ámbito internacional. Además, Kincaid distingue una expansión de las actividades internacionales en las últimas décadas del siglo XX; los estados buscaron promover sus exportaciones y atraer inversión extranjera, debido al estancamiento económico; las acciones 
internacionales de los estados subnacionales se han concentrado principalmente en el comercio y al desarrollo económico.

Cuadro 1. Poderes de las provincias y el gobierno central en Canadá

\begin{tabular}{|c|c|c|}
\hline $\begin{array}{l}\text { Jurisdicción exclusiva de las } \\
\text { provincias }\end{array}$ & $\begin{array}{l}\text { Jurisdicción exclusiva del } \\
\text { gobierno federal }\end{array}$ & $\begin{array}{l}\text { Facultades compartidas } \\
\text { del gobierno federal y } \\
\text { provincial }\end{array}$ \\
\hline Impuestos directos & Paz, orden y buen gobierno & Inmigración \\
\hline $\begin{array}{l}\text { Tierras de la Corona y } \\
\text { recursos naturales }\end{array}$ & $\begin{array}{l}\text { Cualquier forma de impues- } \\
\text { tos }\end{array}$ & Agricultura \\
\hline Hospitales (sector salud) & $\begin{array}{l}\text { Comercio internacional e } \\
\text { interprovincial, comunica- } \\
\text { ciones y transporte }\end{array}$ & Pensiones \\
\hline Educación & Banca y moneda & \\
\hline Prestaciones sociales & $\begin{array}{l}\text { Asuntos exteriores (trata- } \\
\text { dos) }\end{array}$ & \\
\hline Municipalidades & Milicia y defensa & \\
\hline Trabajo local & $\begin{array}{l}\text { Legislación criminal y siste- } \\
\text { ma penitenciario }\end{array}$ & \\
\hline $\begin{array}{l}\text { Negocios y transporte } \\
\text { intra-provincial }\end{array}$ & Naturalización & \\
\hline Administración de justicia & $\begin{array}{l}\text { Pesos, medidas, derechos de } \\
\text { autor y patentes }\end{array}$ & \\
\hline $\begin{array}{l}\text { Derechos civiles y de pro- } \\
\text { piedad. }\end{array}$ & Primeras naciones & \\
\hline \multirow[t]{4}{*}{$\begin{array}{l}\text { Cooperativas y bancos de } \\
\text { ahorro. }\end{array}$} & Poderes residuales & \\
\hline & Poder declarativo. & \\
\hline & Denegación y reservación. & \\
\hline & $\begin{array}{l}\text { Seguro de desempleo y } \\
\text { pensiones por vejez. }\end{array}$ & \\
\hline
\end{tabular}

Fuente: Bélanger (2008).

Es pertinente observar que las tendencias de descentralización que se evidencian en América del Norte también se vienen presentando en América Latina, aunque en menor medida. Naim (2013) advierte que el incremento de países democráticos en América Latina ha contribuido a un mayor nivel 
de descentralización. El número de países de América Latina en los cuales las autoridades ejecutivas de los gobiernos locales (alcaldes) son elegidas directamente por la población, y no designadas por las autoridades centrales, aumentó de 3, en 1980, a 17 en 1995. Además, según Naim, de acuerdo con el Banco Interamericano de Desarrollo, los gobiernos subnacionales de la región pasaron de administrar un 8\% del gasto público en 1990 al 15\% en 2005. En los países más descentralizados, la proporción fue mucho mayor: alrededor de 40\% del gasto del Estado en Argentina, Brasil y Colombia ya no se controla desde el poder central.

En el caso de México, de acuerdo con un estudio de la OCDE (2013), en 2009 los gobiernos de los estados y los municipios en este país ejercieron alrededor del $38 \%$ del total del gasto público, mientras que el gobierno federal concentraba poco más del 60\% de este rubro. De hecho, la proporción del gasto público ejercido por los gobiernos subnacionales ha aumentado en las últimas décadas. Por ejemplo, en 1990, los estados y los municipios en conjunto representaron el 10\% del gasto público en México; este porcentaje aumentó para 2009 al $40 \%$. De acuerdo con este estudio, el incremento en el gasto de los gobiernos subnacionales coincidió con una mayor cantidad de transferencias federales para fines específicos.

No obstante, los procesos de descentralización política no siempre conducen a políticas públicas más eficientes, especialmente en aquellos países que no se han consolidado democráticamente y donde persisten prácticas poco transparentes. Por ejemplo, el proceso de descentralización de las fuerzas policiacas iniciado en México en la década de 1990 no ha generado mayor seguridad en el país. De igual manera, la descentralización del sistema educativo desde finales de la década de 1980, no ha creado un mejor sistema educativo, especialmente en el nivel básico.

De esta manera, el federalismo y la descentralización son factores importantes que dan margen de maniobra a los gobiernos subnacionales para participar en la formulación de políticas públicas y en la conducción de actividades internacionales. Estas tendencias se han expandido en décadas recientes como resultado de la globalización económica neoliberal que ha derivado en una fragmentación del Estado, dando lugar a nuevas formas de gobernanza. En los países de Norteamérica, tales tendencias han sido más evidentes en Estados Unidos y Canadá, mientras que México se ha quedado rezagado por los factores antes expuestos. 


\section{Gobernanza y fragmentación del Estado}

Se puede definir a la gobernanza como "la suma de regulaciones generadas por actores, procesos, así como estructuras y justificada con referencia a un problema público" (Zürn et al, 2010, p. 2). La gobernanza involucra a los actores y procesos que forman parte de un plan de acción, incluyendo negociaciones políticas, construcción de coaliciones, cabildeo (lobbying), persuasión y amenazas que acompañan el proceso de formulación e implementación de políticas públicas. Por otra parte, la investigación sobre gobernanza multi-nivel ha traído consigo un renovado interés en las unidades subnacionales, los departamentos y áreas urbanas. Los niveles subnacionales son vistos como acuerdos "poli-céntricos" de gobernanza o como jurisdicciones funcionales competentes y superpuestas que juegan un rol importante dentro de un contexto multi-nivel (Zürn et al, 2010, p. 7).

Aunque no hay una definición exacta o única, en términos generales la gobernanza se refiere a las diversas formas a través de las cuales se coordina la vida social. La gobernanza por tanto es un proceso (o un complejo de procesos); sus modos principales incluyen mercados, jerarquías, y redes. La gobernanza está representada por una distinción poco clara entre el Estado y la sociedad (organizaciones privadas e instituciones trabajan de manera conjunta con las públicas) y la participación de un número de niveles y capas (potencialmente locales, provinciales, nacionales, regionales y globales). Los procesos a través de los cuales se coordinan los asuntos internacionales son cada vez más identificados como "gobernanza global" (Heywood, 2010, p. 25).

Algunos autores han advertido que el gobierno nacional está cada vez más limitado por un sistema multinivel de gobernanza -local, nacional, regional y global-, que difícilmente puede ser monitoreado, o más bien, controlado. Por ejemplo, Rosenau observa que los problemas globales están gobernados a través de un sistema bifurcado en el cual existen dos mundos de la política global: el primero, un sistema interestatal de Estados y sus gobiernos nacionales que ha dominado por mucho tiempo el curso de los eventos; y el segundo, un sistema multicéntrico de diversos tipos de colectividades que ha surgido recientemente como una fuente opuesta de autoridad, con actores que algunas veces cooperan y otras veces compiten, pero que interactúan continuamente con el sistema Estado-centrista (Rosenau, 2003, p. 225).

Actualmente, de acuerdo con Scholte (2005), la gobernanza involucra regímenes supraestatales (regionales y transnacionales) que operan con cierta autonomía del Estado (p. 186). Además, muchos gobiernos sub-estatales (municipales y provinciales) se involucran directamente con esferas más allá del ámbito nacional. En otras palabras, la gobernanza en el mundo globalizado 
del siglo XXI se ha convertido indistintamente en un mundo entrelazado y multinivel. La regulación ocurre en y a través de conexiones entre los espacios globales, regionales, nacionales, provinciales y municipales. Ningún nivel predomina sobre los otros, como ocurría con la primacía del Estado sobre las esferas subestatales en circunstancias territoriales.

De esta manera, en el mundo globalizado, la sociedad está regulada de una forma poli-céntrica, en la cual, la gobernanza tiende a ser difusa, emanando de múltiples puntos al mismo tiempo, con puntos y líneas de autoridad que no son siempre claros (Scholte, 2005, p. 186). La dispersión de la gobernanza no solo ocurre desde lo local a la global, sino que también se da a través de mecanismos regulatorios en espacios privados junto con los del sector público. Esta situación de multicapas y gobernanza difusa podría ser denominada "policentrismo", para denotar su característica distintiva de emanar de múltiples puntos al mismo tiempo (Scholte, 2005).

Es decir, se ha dado una fragmentación del Estado, con una tendencia descentralizadora que le da más influencia y poder a los gobiernos subnacionales para operar tanto en la arena nacional como en la internacional. Las fuentes de autoridad del mundo multi-céntrico advertido por Rosenau (2006), comprenden a los gobiernos subnacionales, entre otros actores, los cuales se convierten en centros de poder y autoridad en diversas formas. En este sentido, la creciente actividad de los actores subnacionales en la esfera internacional es resultado en gran medida de la reestructuración que ha sufrido el Estado dentro de un contexto de globalización económica y descentralización política.

En este contex to, los gobiernos subnacionales se han convertido en uno de los actores cada vez más relevantes en la gobernanza regional, lo cual se manifiesta en diversas formas de cooperación transnacional. Asimismo, la globalización ha sido acompañada de políticas neoliberales, las cuales han impactado en los gobiernos subnacionales, lo que ha llevado a que éstos busquen fuentes de desarrollo económico en el extranjero. La internacionalización de los gobiernos subnacionales se debe, en parte, a la aplicación de políticas neoliberales, como se muestra en el siguiente apartado.

\section{Globalización y gobiernos subnacionales}

La globalización económica y la gobernanza han reconfigurado el rol de los gobiernos subnacionales de diversas formas. Por ejemplo, John Pierre (2013, p. 17) observa que, durante la década de 1990, la globalización -junto con un régimen neoliberal que propició un desarrollo económico desigual entre las ciudades y regiones-, representó un desafío significativo para los gobiernos subnacionales. 
Subraya que el impacto de la globalización en los estados, regiones y ciudades ha llegado a ser cada vez más articulado; este impacto ha sido más fuerte y directo en las economías e instituciones subnacionales, que en las economías políticas nacionales.

Además, resalta Pierre, la globalización ha coincidido con, y frecuentemente propiciado, procesos importantes de cambio institucional doméstico. Las políticas económicas del Estado en las democracias occidentales durante las décadas de 1980 y 1990 han enfatizado de manera creciente objetivos neoliberales, tales como reducción de impuestos, desregulación y recortes en el sector público. Estas políticas tienden a dejar a las ciudades y a las regiones menos protegidas de las presiones económicas y políticas internacionales, a diferencia del tipo de políticas públicas que prevalecía anteriormente, por ejemplo, mayores impuestos, mayor regulación, un sector público más interventor y con mayores recursos. En este mismo sentido, los subsidios del gobierno central a los gobiernos subnacionales eran anteriormente más constantes y generosos. Estos estímulos tenían como fin compensar a las ciudades y regiones para la implementación de políticas públicas nacionales.

El efecto combinado de las políticas neoliberales del gobierno central y la reducción de los subsidios de los gobiernos centrales a los gobiernos subnacionales, ha llevado a que muchas ciudades y regiones estén explorando otras fuentes de ingresos y desarrollo económico, incluyendo redes en el extranjero $^{5}$. La era de la globalización ha propiciado una restructuración institucional, generando procesos como la internacionalización subnacional, y la emergencia de acuerdos de gobernanza regionales o transnacionales. Asimismo, la internacionalización subnacional está claramente vinculada a la globalización, incentivando a las ciudades y regiones a buscar alianzas estratégicas en el extranjero e indirectamente a través de la política neoliberal de estimular la competencia entre ciudades y regiones (Pierre, 2013).

De igual manera, Lachapelle y Paquin (2005) advierten que la víctima de la globalización es el Estado-nación, cuya capacidad para regular la economía

\footnotetext{
4. Las políticas neoliberales fueron identificadas por John Williamson hacia finales de la década de los 1980s; y representan de manera específica lo que se conoce como el Consenso de Washington: (1) Disciplina fiscal, (2) La inflación como parámetro central de la economía, (3) Prioridades en el gasto público, (4) Reforma Tributaria, (5) Tasas de interés, (6) Tipo de cambio, (7) Política comercial, (8) Inversión Extranjera Directa (IED), (9) Privatizaciones, (10) Desregulación. Para mayor detalles del neoliberalismo, véase: Roberto Zepeda (2013), Neoliberalismo, desempeño económico y mercados laborales en Latinoamerica: un enfoque comparativo, Ánfora, vol. 20, núm. 35, julio-diciembre, pp. 13-40.

5. Para mayores detalles sobre cómo la globalización ha afectado a los gobiernos subnacionales y los ha motivado para expandir sus actividades en la arena internacional, véase: Jon Pierre (2013). Globalization and governance. Edward Elgar Publishing.
} 
nacional se ha reducido significativamente. Para estos autores, la autoridad se fragmenta entre los diferentes actores, públicos y privados, en el escenario internacional, nacional, regional y local. La globalización está cambiando las reglas del juego para todos los jugadores y esta situación está creando espacio para nuevas estrategias, nuevos métodos de funcionamiento y nuevas posibilidades de gobernanza.

Considerando lo anterior, es evidente que los efectos de la globalización han socavado la capacidad del gobierno para responder a un número cada vez mayor de asuntos de política públicas. Como resultado, los gobiernos subnacionales se han visto en desventaja y han tenido que buscar otras formas de desarrollo económico en el ámbito internacional. Diversos autores han conceptualizado las relaciones internacionales de los gobiernos subnacionales como paradiplomacia. La siguiente sección analiza las diferentes acepciones de este término, sus formas y características, en especial en Norteamérica. La paradiplomacia es un elemento consustancial a la gobernanza regional en Norteamérica. Es decir, se crean espacios de gobernanza a través de la paradiplomacia.

\section{Paradiplomacia}

Como ha sido mencionado, los gobiernos subnacionales han visto un aumento de su poder e influencia en un contexto de descentralización y fragmentación del Estado, creando nuevas formas de gobernanza regional. Es precisamente en estas condiciones, en las que los gobiernos subnacionales han aumentado sus actividades internacionales. De acuerdo con Kuznetsov (2015), se denomina paradiplomacia a la participación o involucramiento de las unidades constituyentes (regiones) de los Estados nacionales en asuntos internacionales, como las provincias en Canadá, los estados en Estados Unidos, las comunidades autónomas en España, las landers en Alemania, los oblasts y las repúblicas en Rusia.

En este sentido, los gobiernos regionales desempeñan actividades de relaciones internacionales de diferentes maneras: “establecen misiones comerciales y culturales en el extranjero; firman tratados y acuerdos con actores internacionales estatales y no-estatales; participan en redes internacionales de cooperación regional, y en ocasiones cuestionan la política exterior oficial de sus gobiernos centrales por medio de discursos o acciones" (Kuznetsov 2015, p. 3).

Para Noe Cornago (2013), la paradiplomacia involucra la participación de los gobiernos no centrales en las relaciones internacionales mediante el establecimiento de contactos permanentes o ad hoc con entidades públicas o privadas extranjeras, con el propósito de promover cuestiones socioeconómicas 
o culturales, así como cualquier otra dimensión externa de sus competencias constitucionales.

Ivo Duchacek (1990), uno de los primeros autores en utilizar este término, identifica tres formas diferentes de paradiplomacia. En primer lugar, la paradiplomacia regional transfronteriza se refiere a los contactos transfronterizos -institucionales, formales, y sobre todo, informales- que están condicionados por la proximidad geográfica y la naturaleza de problemas comunes y sus posibles soluciones. En segundo lugar, la paradiplomacia trans-regional se refiere a las conexiones y negociaciones entre los gobiernos no-centrales que no son vecinos, pero cuyos gobiernos nacionales sí lo son. Finalmente, la paradiplomacia global consiste en los contactos políticos-funcionales con naciones distantes que conectan a los gobiernos no centrales no sólo con centros comerciales, industriales, o culturales en otros continentes, sino también con las distintas ramas o agencias de los gobiernos nacionales extranjeros (Duchacek, 1990, pp. 18-27).

Algunos autores, por ejemplo, Nossal, Rousell y Paquin (2015) distinguen entre la paradiplomacia y la protodiplomacia. La paradiplomacia se refiere a las actividades internacionales de un gobierno no-central que son conducidas junto con la diplomacia del gobierno central. En contraste, la protodiplomacia es implementada por un gobierno no-central que busca utilizar sus actividades internacionales para ganar reconocimiento de otros Estados y de esta manera transformar la protodiplomacia en diplomacia pura y simple, mediante el logro de independencia y soberanía al nivel de categoría de Estado. En el caso de Quebec, se han visto episodios esporádicos de protodiplomacia -a finales de los 1970 y a mediados de los 1990s, por ejemplo-. Sin embargo, actualmente, en general las actividades internacionales del gobierno provincial se han dirigido a asegurar no la independencia para Quebec como Estado soberano, sino una paradiplomacia que fortalezca la identidad de Quebec (Nossal et al., 2015).

Respecto al caso de Canadá, Vengroff y Rich (2006) sostienen que el aumento de la paradiplomacia es el resultado de diversos factores clave, tales como: "la creciente globalización, los acuerdos comerciales internacionales (...), el impacto actual del federalismo, el nacionalismo, la descentralización, las disposiciones constitucionales (un tanto ambiguas) vigentes y la expansión de la actividad internacional en esferas reservadas para las unidades subnacionales" (pp. 106107) son algunos de los factores más importantes.

En el caso de los Estados Unidos, se ha dado un incremento en las actividades internacionales de los estados en las últimas dos décadas. Por ejemplo, solo 4 estados de Estados Unidos tenían representaciones permanentes en el exterior en 1980; mientras que para el año 2002, este número aumentó a casi 40 de los 50 
estados de este país; de esta manera, el número de este tipo de oficinas aumento de 151 en 1992 a 240 en el año 2000 (Scholte, 2005, p. 205).

Considerando lo anterior, se puede remarcar que las actividades diplomáticas tradicionales, conducidas por los gobiernos nacionales, no han sido suficientes para resolver los problemas de la región. Problemas globales como la migración, seguridad, contaminación, cambio climático, entre otros, no están siendo afrontados de manera efectiva desde los ámbitos nacionales. Como resultado, los estados subnacionales se han convertido en avenidas de soluciones y propuestas para los problemas globales más graves y complejos de principios del siglo XXI.

La siguiente sección se enfoca en la paradiplomacia de las provincias canadienses, precisamente debido a que, como hemos señalado, Canadá es el país donde estas tendencias se presentan con mayor relevancia debido a un sistema político federal altamente descentralizado.

\section{Dimensiones de la paradiplomacia de las provincias canadienses}

En Canadá, los gobiernos provinciales generalmente son libres de actuar internacionalmente dentro de sus áreas de competencia descritas en la constitución canadiense, y se espera que el gobierno nacional consulte con las provincias antes de entrar a cualquier tratado internacional que afecte las provincias en sus áreas de competencia (Fry, 2011). Todas las provincias canadienses han desarrollado relaciones con los estados vecinos de Estados Unidos para resolver problemas en común y administrar recursos, así como para promover intercambios económicos y atraer inversión.

En otras regiones del mundo también se observan dinámicas similares. Fry (2011) resalta que la región flamenca en Bélgica; Cataluña y el País Vasco en España; y Quebec en Canadá, tienen los programas más activos de relaciones internacionales, en términos de dinero sufragado y personal dedicado a las actividades internacionales. La provincia canadiense con mayor nivel de relaciones internacionales es Quebec, seguida por Alberta, Ontario y Columbia Británica.

De acuerdo con Lecours (2009: 127), la actividad internacional de las provincias de Canadá es principalmente funcional, ya que responde a intereses económicos, para la promoción de exportaciones y la atracción de inversión extranjera, así como compartir información y en ocasiones coordinar políticas públicas, con los estados vecinos de Estados Unidos. De hecho, los gobiernos provinciales de Canadá gastan más en programas internacionales y tienen más personal involucrado en tales programas que los 50 estados de los Estados Unidos en conjunto, aun cuando las provincias representan colectivamente una 
novena parte en términos de población y su Producto Interno Bruto es solo una catorceava parte del de Estados Unidos (Fry, 2004, p. 12). De igual manera, algunas ciudades canadienses como Montreal (Quebec), Vancouver (Columbia Británica) y Toronto (Ontario) también han desarrollado una dimensión internacional (Lecours, 2009, p. 128).

Vengroff y Rich (2006) destacan que Quebec ha sido la provincia más activa en lo concerniente a actividades paradiplomáticas (p. 119). Estos autores subrayan la dimensión de la paradiplomacia de Quebec: esta provincia cuenta con un departamento con personal dedicado exclusivamente a las relaciones internacionales. Desde 1964, Quebec ha firmado más de 550 acuerdos internacionales, de los cuales más de 300 todavía están vigentes, con 79 países diferentes. Vengroff y Rich también remarcan que esta provincia tiene una considerable presencia en el exterior, con una red de 28 oficinas en 17 países incluyendo, los Estados Unidos- en Latinoamérica, Europa y Asia (2006, p. 119). Además, Quebec ha creado su propio ministerio de relaciones internacionales con más de 550 empleados y un presupuesto anual que sobrepasa los 100 millones de dólares (Fry, 2011).

Por otra parte, es evidente el involucramiento de los gobiernos subnacionales en el ámbito internacional entre los países de Norteamérica; sin embargo, es pertinente observar que en algunos casos estas relaciones se han institucionalizado entre estados transfronterizos de los países de la región, creando foros y conferencias, que se convierten en espacios periódicos de cooperación transnacional. Existen diversos esquemas de cooperación transnacional entre estados transfronterizos de la región que es pertinente observar. La siguiente sección se enfoca precisamente en los mecanismos de interacción entre los gobiernos subnacionales de la región, en particular de la región fronteriza de Canadá y Estados Unidos y de Estados Unidos y México.

\section{Paradiplomacia transfronteriza en América del Norte}

En un contexto político, caracterizado por sistemas federales de gobierno descentralizados, el TLCAN ha ayudado a fortalecer los vínculos políticos e institucionales entre las provincias de Canadá y los estados de México y Estados Unidos. Se han creado diversas organizaciones transfronterizas para facilitar una mayor cooperación regional entre gobiernos no-centrales.

Algunos estados fronterizos en América del Norte que enfrentan problemáticas comunes han formado foros y grupos de trabajo permanentes; por ejemplo, los 4 estados del sur de Estados Unidos (California, Arizona, Nuevo México y Texas) y los 6 del norte de México (Baja California, Sonora, Chihuahua, Coahuila, 
Nuevo León, y Tamaulipas) han realizado reuniones conjuntas de manera periódica para signar acuerdos de cooperación sobre temas tan variados como: desarrollo económico, puertos comerciales, educación, salud y seguridad, entre otros (Embajada de México en Estados Unidos, 2012). Este foro de cooperación se conoce como la Conferencia de Gobernadores Fronterizos.

La primera conferencia de gobernadores fronterizos se realizó en Ciudad Juárez, Chihuahua, México en 1980, la cual dio inicio a un proceso formal de apertura de líneas de comunicación entre los 10 estados fronterizos de Estados Unidos y México. Se llevan a cabo conferencias periódicas, alternando las sedes entre Estados Unidos y México (Arizona Government, 2016). Otros ejemplos de cooperación entre estados subnacionales de estos dos países incluyen la Comisión México -Arizona, la Conferencia de Gobernadores Fronterizos, la Conferencia de Legisladores Fronterizos, la Comisión Fronteriza ChihuahuaNuevo México, y la Comisión de las Californias (McMillan, 2012).

De igual manera, existen varios espacios en los que las relaciones entre gobiernos subnacionales en los Estados Unidos y Canadá se han institucionalizado. Samuel McMillan (2012) observa que la globalización económica y comercial acrecentó los vínculos transfronterizos de aquellos estados de EE.UU. que colindan con Canadá o México, involucrándolos en asuntos tan variados como el comercio, la seguridad fronteriza, inmigración y problemas del medio ambiente. Se han creado organizaciones transfronterizas, tales como la Comisión de los Gobernadores de Nueva Inglaterra y los Primeros Ministros del Este de Canadá, el Consejo de Gobernadores de Los Grandes Lagos (CGLG por sus siglas en inglés), Fuerza Especial Idaho -Alberta, Consejo Consultivo Bilateral Montana- Alberta, la Región Económica Pacifico Noroeste, y la Asociación de los Gobernadores del Oeste y los Primeros Ministros Canadienses del Oeste (McMillan, 2012, p. 78). A continuación, se describen algunas de estas organizaciones regionales.

a) La Conferencia de Gobernadores y Premieres de los Grandes Lagos y San Lorenzo.

Esta conferencia se fundó el año de 1983; está integrada por los siguientes miembros: en Estados Unidos, los estados de Illinois, Indiana, Michigan, Minnesota, Nueva York, Ohio, Pennsylvania y Wisconsin. En Canadá, las provincias de Ontario y Quebec. A través de la Conferencia, los Gobernadores y Premieres trabajan para hacer crecer la economía de la región, estimada en 5 billones de dólares y proteger el sistema más grande de agua fresca en el mundo 
(CGLSLGP, 2016) ${ }^{6}$. La Conferencia es resultado de más 30 años de trabajo del Consejo de los Grandes Lagos para estimular y facilitar un desarrollo económico responsable con el medio ambiente.

b) La Conferencia de Gobernadores de Nueva Inglaterra y Premieres del Este de Canadá.

La Conferencia de Gobernadores de Nueva Inglaterra y los Premieres del Este de Canadá representa gran parte de la actividad transfronteriza en la región. Como uno de los mecanismos regionales transfronterizos más antiguos, esta conferencia se estableció en 1976 y ha dedicado especial atención a temas como desarrollo sustentable y medio ambiente. Asimismo, los estados miembros han formulado una serie de acciones para reducir la contaminación del aire y los gases de efecto invernadero ${ }^{7}$. Sus miembros fundadores están conformados por 11 gobiernos subnacionales: 5 Provincias de Canadá (Nueva Brunswick, Terranova y Labrador, Nueva Escocia, Isla del Príncipe Eduardo y Quebec) y 6 estados de Estados Unidos (Connecticut, Maine, Massachusetts, New Hampshire, Rhode Island, Vermont).

c) Alianza entre estados del Sudeste de Estados Unidos y las Provincias Canadienses (SEUS-CP).

La alianza estratégica entre los estados del sudeste de Estados Unidos y las Provincias de Canadá fue establecida en 2007 en Montreal, Quebec, con el objetivo de promover las oportunidades de comercio e inversión entre los estados miembros y las provincias. Los estados miembros incluyen Alabama, Georgia, Mississippi, Carolina del Norte, Carolina del Sur y Tennessee de Estados Unidos y las provincias de Manitoba, Nueva Brunswick, Terranova y Labrador, Nueva Escocia, Ontario, Isla del Príncipe Eduardo y Quebec de Canadá. La Alianza se reúne en una conferencia anual encabezada por los gobernadores de cada estado, y los Premieres o sus representantes (líderes empresariales e industriales). La conferencia permite a los líderes del sudeste de Canadá y de Estados Unidos negociar con una red de líderes del sector público y privado. Los temas más relevantes abordados son: nuevas tecnologías de la comunicación, manufactura avanzada y tecnología limpia (SEUS-CP, 2016).

\footnotetext{
6. Para mayor información sobre esta conferencia, véase el portal: http//:www.cglslgp.org

7. Para mayor detalle sobre esta conferencia, véase Robert G. Healy, Debora Van Nijnatten, y Marcela López-Vallejo. 2014. Environmental policy in North America: Approaches, capacity, and the management of transboundary issues. University of Toronto Press.
} 
d) Consejo de Gobiernos Estatales -Conferencia Regional del Este (CSGERC).

El propósito de la Conferencia Regional del Este (CSG-ERC por sus siglas en inglés) es facilitar el intercambio de ideas entre los formuladores de políticas públicas, líderes empresariales y la comunidad académica de los 18 estados miembros. Estos gobiernos subnacionales incluyen los 11 estados del noreste de EE.UU. desde Maine a Maryland, Puerto Rico, y las Islas Vírgenes; y en Canadá, las provincias de Quebec, Nueva Brunswick, Ontario, Nueva Escocia y la Isla del Príncipe Eduardo. La oficina sede de esta conferencia está situada desde 1937 en la ciudad de Nueva York.

Durante más de 70 años, los miembros de esta conferencia han tratado de capacitar a los funcionarios públicos en relación con los problemas y desafíos que enfrenta la región. Al facilitar la cooperación entre sus miembros, este foro promueve soluciones multi-estatales o regionales a los problemas comunes. En cumplimiento de su misión, se llevan a cabo investigaciones y análisis de políticas públicas; se organizan seminarios y conferencias y se publican boletines electrónicos relacionados con temas variados como agricultura, energía/medio ambiente, salud, transporte y tendencias regionales.

Recientemente se reunieron los Premieres de Canadá con los gobernadores de Estados Unidos y México. En 2015, se realizó la Primera Cumbre de Gobernadores y Premieres de América del Norte, en el estado de Colorado, Estados Unidos. Participaron siete gobernadores de Estados Unidos, seis de México y dos de Canadá. El objetivo fue promover el diálogo transfronterizo en búsqueda de actuaciones y oportunidades conjuntas en materia de economía, política, energía, desarrollo sostenible y medio ambiente.

Por parte de México, asistieron los gobernadores de seis estados (Hidalgo, Jalisco, Puebla, Quintana Roo, Yucatán y México) en representación de la Conferencia Nacional de Gobernadores (CONAGO). Los gobernadores estadounidenses de Colorado, Utah, Michigan, Dakota del Sur, Nuevo México, Islas Vírgenes e Illinois, participaron como miembros de la Asociación Nacional de Gobernadores de Estados Unidos (NGA); y los premieres canadienses de Yukón y Nueva Brunswick participaron como representantes del Consejo de la Federación de Canadá, COF por sus siglas en inglés (National Governors Association, 2015). Es decir, se trató de una reunión conjunta de las tres asociaciones que congregan a las autoridades subnacionales de los tres países que conforman la región de América del Norte.

Considerando lo anterior, se puede señalar que las relaciones internacionales de los estados subnacionales entre países de América del Norte no se concentran solo entre estados fronterizos (paradiplomacia regional transfronteriza), sino 
que también se han creado organizaciones y foros de cooperación entre estados subnacionales distantes, pero de países vecinos (paradiplomacia transregional); además, hay otros casos de relaciones internacionales entre estados subnacionales de países distantes (paradiplomacia global). En los últimos años ha crecido el número de oficinas internacionales de las provincias canadienses en países de Asia, especialmente en China.

Una revisión de las características de las relaciones paradiplomáticas en los casos antes analizados, permite evidenciar la participación no solo de los gobiernos subnacionales (es decir de gobernadores y premieres) en las organizaciones regionales, sino también de líderes empresariales e industriales, así como una red de líderes del sector público y privado y la comunidad académica, con el objetivo de tomar decisiones conjuntas. De igual manera, se percibe la influencia de organizaciones no gubernamentales en los temas cubiertos en conferencias y organizaciones, entre los que destacan el medio ambiente y desarrollo sustentable.

Como se mostró, la Conferencia de Gobernadores Fronterizos es el caso más relevante de paradiplomacia regional transfronteriza entre Estados Unidos y México. Sin embargo, las reuniones en el marco de esta conferencia han disminuido en años recientes. No obstante, es pertinente subrayar que la cooperación entre algunos estados fronterizos se ha intensificado de forma bilateral; destaca el caso de Arizona y Sonora, los cuales tienen 14 acuerdos de cooperación interinstitucional sobre diversos temas como desarrollo económico, laboral, seguridad, turismo, tráfico de armas, y de personas, entre otros (SRE, 2016).

\section{Conclusions}

Diversos estados subnacionales han incrementado sus actividades internacionales con el fin de promover sus intereses en el exterior, principalmente la promoción del comercio y la atracción de la inversión extranjera; además, han buscado acuerdos de cooperación en áreas como medio ambiente y desarrollo sostenible, entre otras. La mayoría de los acuerdos y políticas que se han concretado se circunscriben a las áreas de jurisdicción de los gobiernos subnacionales y, al mismo tiempo, revelan cierto grado de autonomía de éstos con respecto al gobierno central.

Las formas específicas de las actividades internacionales de los gobiernos subnacionales se concentran principalmente en el establecimiento de oficinas de representación en el extranjero; asimismo, se han signado acuerdos de cooperación con otros estados de la región. La relevancia de las actividades internacionales de gobiernos subnacionales es crucial, ya que muchos de los 
problemas locales se están abordando en estos espacios transnacionales de cooperación entre actores subnacionales de distintos países.

La creciente integración económica en América del Norte ha facilitado otras formas de integración a través de las acciones emprendidas por los gobiernos subnacionales. Lo anterior ha ocurrido debido a la fragmentación del poder del gobierno central, lo que ha conducido al surgimiento de diversas fuentes de autoridad, tanto en el ámbito nacional e internacional. Dentro de la nueva gobernanza de la región, destaca la participación de los gobiernos subnacionales en espacios de cooperación transnacional con otras unidades subnacionales, que en ocasiones se convierten en foros institucionales. Estos foros han congregado a estados y provincias de Estados Unidos y Canadá con el fin de coordinar los esfuerzos para enfrentar desafíos comunes en diversas áreas.

La mayoría de las actividades internacionales de los estados subnacionales, tales como acuerdos y otros esquemas de cooperación se ubican en el ámbito de sus atribuciones constitucionales. No obstante, en algunos casos los gobiernos subnacionales persiguen objetivos independientes de los gobiernos subnacionales, en especial aquellas provincias que se consideran como una nación dentro de un Estado, como por ejemplo el caso de Quebec en Canadá, aunque en años recientes esta tendencia ha disminuido.

Finalmente, es evidente que los gobiernos subnacionales contribuyen de manera relevante a la gobernanza de América del Norte a través de la formulación de políticas públicas regionales para afrontar los retos comunes que enfrentan los estados subnacionales de una región. Muchos de estos acuerdos se circunscriben en las áreas de jurisdicción de los gobiernos subnacionales y revelan un cierto grado de autonomía respecto al gobierno central.

Algunos de los casos de cooperación entre gobiernos subnacionales, analizadas aquí, presentan esquemas novedosos que pueden servir de ejemplo para otros países de América Latina que forman parte de bloques regionales como el Mercosur, entre otros, que se han convertido en contextos propicios para la cooperación entre gobiernos subnacionales de esta región.

\section{References}

Arizona Government (2016). Border Governors Conference. Recuperado de: https://tourism.az.gov/border-governors-conference.

Balthazar, L. (1999). The Quebec experience: Success or failure? Regional \& $\mathrm{Fe}$ deral Studies, 9(1): 153-169. 
Bélanger, C. (2008). Canadian Federalism -Division of Powers (ss. 91-95 of the Constitution Act 1867). Marianopolis College. Recuperado de: http://faculty.marianopolis.edu/c.belanger/QuebecHistory/readings/Canadadivisionofpowers.html

Burgess, M. (2006). Comparative federalism: Theory and practice. Londres-Nueva York: Routledge.

Curzio, L. (2000). El federalismo mexicano: balance y perspectivas. En G. Ibarra y A. L. Ruelas (eds.). México y Canadá en la globalización: fronteras tensionadas en América del Norte (pp. 257-280), México: Porrúa.

Conference of Great Lakes and St. Lawrence Governors and Premiers (CGLSLGP), (2016). Recuperado de: http://www.cglslgp.org/

Cornago, N. (2013). Diplomacy and Paradiplomacy in the Redefinition of International Security: Dimensions of Conflict and Cooperation. En Aldecoa, F. (Ed.) Paradiplomacy in Action: The Foreign Relations of Subnational Governments (pp. 40-57), Londres-Nueva York: Routledge.

The Council of State Governments Eastern Regional Conference (CSG/ERC). Recuperado de: http://www.csg-erc.org/

Duchacek, I. (1990). Perforated Sovereignties: Towards a Typology of New Actors in International Relations. En Hans J. Michelmann y Panayotis Soldatos (Eds.). Federalism and International Relations: The Role of Sub-national Units. Oxford: Clarendon Press.

Embajada de México en Estados Unidos (2012). El Embajador Arturo Sarukhán participa en XXX Conferencia de Gobernadores Fronterizos en Albuquerque, Nuevo Mexico. Recuperado de: http://embamex.sre.gob. $\mathrm{mx} / \mathrm{eua} /$ index.php/es/comunicados2012/591-el-embajador-arturo-sarukhan-participa-en-xxx-conferencia-de-gobernadores-fronterizos-en-albuquerque-nuevo-mexico, consultado en octubre de 2014.

Fry, E. H. (2011). Federalism and Foreign Relations. En George T. Kurian, James E. Alt, Simone Chambers, Geoffrey Garret, Margaret Levi, Paula D. Mcclain (Eds.) The Encyclopedia of Political Science. California: CQPress (pp. 573-574).

Fry, E. H. (2004). The Role of Sub-National Governments in North America Integration. Thinking North America. Canada: Institute for Research on Public Policy.

Fry, E. H. (2005). Sub-State strategies in an era of globalization and the information technology revolution. En S. Paquin y G. LaChappelle (Eds.). Mastering globalization: new sub-states' governance and strategies (pp. 116-123). Londres-Nueva York: Routledge. 
Hale, G. (2012). So near yet so far: The public and hidden worlds of Canada-US relations. Canada: UBC Press.

Healy, R. G. et al. (2014). Environmental policy in North America: Approaches, capacity, and the management of transboundary issues. University of Toronto Press.

Heywood, A. (2010). Global politics. Basingstoke: Palgrave Macmillan.

Hague, R. y Harrop, M. (2004). Comparative government and politics. Londres: Palgrave Macmillan.

Hocking, B. (1993). Localizing foreign policy: Non-central governments and multilayered diplomacy. Springer.

Kincaid, J. (2013). The international competence of US states and their local governments" en F. Aldecoa (ed.), Paradiplomacy in action: the foreign relations of subnational governments, Londres - Nueva York: Routledge, pp. 111-133.

Kuznetsov, A. (2014) Theory and Practice of Paradiplomacy. Londres - Nueva York: Routledge.

Kukucha, Ch. J. (2008). The provinces and Canadian foreign trade policy. Vancouver: UBC press.

Lecours, A. (2009). Canada. En Hans Michelmann (Ed.) Foreign Relations in Federal Countries (pp. 114-139). Montreal y Kingston -Londres, Ithaca: McGill-Queen's University Press, Forum of Federations

Márquez-Padilla, P.C. (2012), Justicia Internacional: ideas y reflexiones, México, CISAN-UNAM.

Pierre J. (2013). Globalization and governance. Edward Elgar Publishing.

McHugh, J. (2015). Paradiplomacy, protodiplomacy and the foreign policy aspirations of Quebec and other Canadian provinces", Canadian Foreign Policy Journal, 21(3), 238-256.

McMillan, S. L. (2012). The Involvement of State Governments in U.S. Foreign Relations. Nueva York: Palgrave Macmillan.

Naím, M. (2013). El fin del poder. Bogotá: Debate.

National Governors Association, (2015). North American leaders meet for firstever Summit. Recuperado de: http://www.nga.org/cms/home/news$\mathrm{room} /$ news-releases/2015--news-releases/col2-content/north-american-leaders-summit.html

Nossal, K. R. et al (2015). The politics of Canadian foreign policy. McGill-Queen's Press-MQUP.

OCDE (2013) Estudios Económicos de la OCDE: México 2013, Centro de la OCDE para América Latina. 


\section{OECD (2015) Government at a Glance 2015. Paris: OECD.}

Paquin, S. (2010). Federalism and compliance with international agreements: Belgium and Canada compared. The Hague Journal of Diplomacy 5(1-2), 173-197.

Paquin, S. y LaChappelle, G. (2005). Introduction. En S. Paquin y G. LaChappelle (Eds.). Mastering globalization: new sub-states' governance and strategies. Londres-Nueva York: Routledge.

Rosenau, J. N. (2003). Governance in a new global order. En D. Held y A. McGrew (Eds.). Governing Globalization: Power, Authority and Global Governance (pp. 70-86). Cambridge-Malden: Polity Press.

Rosenau, J. N. (2006). The Study of World Politics: Globalization and Governance. Londres - Nueva York: Routledge.

Scholte, J. A. (2005) Globalization: a critical introduction. Houndmills Basingstoke -Nueva York: Palgrave MacMillan.

Secretaria de Relaciones Exteriores (SRE) (2014). América del Norte del Siglo XXI: construyendo la región más Dinámica y Competitiva del Mundo. Recuperado de: http://saladeprensa.sre.gob.mx/index.php/es/comunicados/3728-050

Secretaria de Relaciones Exteriores (SRE) (2016). Gobiernos Locales. Recuperado de: http://www.sre.gob.mx/coordinacionpolitica/index.php/diplomacia-local

Southeastern United States -Canadian Provinces Alliance (SEUS-CP) (2016). REcuperado de: http://seuscp.com/us/

United Nations for Development Programme (UNDP) (2004). Decentralized governance for development. A combined practice note on decentralization, local governance and urban / rural development. Nueva York: UNDP.

United States Embassy in Mexico, (2014). Bilateral Trade fact-Sheet. http:// photos.state.gov/libraries/mexico/310329/july2014/2014_07_Bilateral\%20Trade.pdf

Vengroff, R. y Rich, J. (2006). Foreign policy by other means: Paradiplomacy and the Canadian provinces. En P. James, N. Michaud y M. J. O’Reilly (Eds.). Handbook of Canadian Policy (pp. 105-130). Toronto: Lexington Books.

Zürn, M. et al. (2010). Introduction. En H. Enderlein, S. Wälti y M. Zürn (Eds.). Handbook on multi-level governance (pp. 1-13). Cheltenham: Edward Elgar.

Zepeda, R. (2013). Neoliberalismo, desempeño económico y mercados laborales en Latinoamérica: un enfoque comparativo. Ánfora, 20(35), 13-40. Universidad Autónoma de Manizales. 